\title{
Cross-Clamp Technique and Incidence of Stroke after Coronary Artery Bypass Grafting: Know and Respect the Aorta Principle
}

\author{
Giorgio Mastroiacovo ${ }^{1}$, Marco Gennari ${ }^{1}$, Ilaria Giambuzzi ${ }^{2}$, Sergio Pirola ${ }^{3}$, Giuseppe \\ Nanci $^{3}$, Alice Bonomi ${ }^{3}$, Gabriele Egidy Assenza ${ }^{4}$, Gianluca Polvani ${ }^{5}$, and Marco Agrifoglio ${ }^{6}$ \\ ${ }^{1}$ Centro Cardiologico Monzino IRCCS \\ ${ }^{2}$ Affiliation not available \\ ${ }^{3}$ Centro Cardiologico Monzino Istituto di Ricovero e Cura a Carattere Scientifico \\ ${ }^{4}$ IRCCS Azienda Ospedaliero-Universitaria di Bologna Policlinico S Orsola-Malpighi \\ Dipartimento cardio-toracico-vascolare \\ ${ }^{5}$ centro cardiologico monzino \\ ${ }^{6}$ Centro Cardiologico Monzino
}

February 17, 2021

\begin{abstract}
Objectives: One of the most severe and devastating complications following coronary artery bypass grafting (CABG) are cerebro-vascular accidents. Atherosclerotic disease of the ascending aorta and epi-aortic trunks has been considered the most probable cause of cerebral embolization during CABG due to aortic manipulation and clamping. The aim of this study is to investigate if single or double aortic clamping may impact the incidence of neurological events. Methods: This is a retrospective study which includes a series of patients who underwent CABG from a single surgeon at our Institution from March 2006 to December 2012. Patients were divided into two homogenous groups based on the surgical technique: single-aortic clamping (SAC) (118 patients) and double aortic clamp (DAC) (133 pt). Results: Median surgical time was longer for the DAC group than for the SAC group $(\mathrm{p}=0.015)$, but no significant differences were found for the primary outcomes of stroke and transient ischemic attack. The two groups presented a similar 30-day survival. The follow-up was completed at $82 \%$ (median 11 years). The Kaplan-Meier estimates a survival at 11 years of $81 \%$ and $88 \%$ for the DAC and SAC group, respectively. Conclusions: Incidence of stroke seems to be independent from cross-clamping technique and we could infer that the global rate of stroke after CABG in low to moderate risk patients is probably more influenced by other factors that further studies need to address. Moreover, the appearance of the aorta at the time of surgery is crucial to analyse to better personalize the strategy.

Cross-Clamp Technique and Incidence of Stroke after Coronary Artery Bypass Grafting: Know and Respect the Aorta Principle

Giorgio Mastroiacovo ${ }^{1 *}$, Marco Gennari ${ }^{*}$, Ilaria Giambuzzi ${ }^{1}$, Sergio Pirola ${ }^{1}$, Giuseppe Nanci ${ }^{1}$, Alice Bonomi $^{2}$, Gabriele Egidy Assenza ${ }^{3}$, Gianluca Polvani ${ }^{4}$ and Marco Agrifoglio ${ }^{1,5}$

*these authors contributed equally to this work

${ }^{1}$ Department of Cardiovascular Surgery, IRCCS Centro Cardiologico Monzino, Milan, Italy

${ }^{2}$ Department of Statistics, IRCCS Centro Cardiologico Monzino Milan, Italy

${ }^{3}$ Departement of Pediatric Cardiology and Adult Congenital Heart Disease, Sant'Orsola Malpighi Hospital, Bologna, Italy

${ }^{4}$ Department of Cardiovascular Sciences and Community Health, University of Milan, Italy
\end{abstract}


${ }^{5}$ Department of Biomedical, Surgical and Dental Sciences, University of Milan, Italy

Word count: 4500

Corresponding author:

Giorgio Mastroiacovo MD, IRCCS Centro Cardiologico Monzino,Via Parea 4-20138 Milan,Italy

E-mail:gio.mastroiacovo@hotmail.it

Phone:+393337043598.

Abstract

Objectives: One of the most severe and devastating complications following coronary artery bypass grafting $(\mathrm{CABG})$ are cerebro-vascular accidents. Atherosclerotic disease of the ascending aorta and epi-aortic trunks has been considered the most probable cause of cerebral embolization during CABG due to aortic manipulation and clamping. The aim of this study is to investigate if single or double aortic clamping may impact the incidence of neurological events.

Methods : This is a retrospective study which includes a consecutive series of patients who underwent CABG from a single CABG-experienced surgeon at our Institution from March 2006 to December 2012. Patients were divided into two homogenous groups based on the surgical technique adopted: single-aortic clamping (SAC) (118 patients) and double aortic clamp (DAC) (133 pt).

Results : Median surgical time was statistically longer for the DAC group than for the SAC group $(\mathrm{p}=$ 0.015), but no significant differences were found for the primary outcomes of stroke and transient ischemic attack. The two groups presented a similar 30-day survival. The follow-up was completed at $82 \%$ (median 11 years). The Kaplan-Meier estimates a survival at 11 years of $81 \%$ and $88 \%$ for the DAC and SAC group, respectively.

Conclusions : Incidence of stroke seems to be independent from cross-clamping technique and, more generally, we could infer that the global rate of stroke after CABG in low to moderate risk patients is probably more influenced by other factors that further studies need to address. Moreover, the appearance of the aorta at the time of surgery is crucial to analyse to better personalize the strategy.

Keywords: coronary artery bypass artery, stroke, coronary artery disease, neurological complications

List of abbreviations:

$\mathrm{CABG}=$ Coronary Artery Bypass Grafting

$\mathrm{CPB}=$ Cardiopulmonary Bypass

$\mathrm{DAC}=$ Double Aortic Clamp

$\mathrm{EF}=$ Ejection Fraction

LIMA = Left Internal Mammary Artery

MACCE $=$ Major Adverse Cerebral and Cardiovascular Events

OPCAB $=$ Off-pump Coronary Artery Bypass

$\mathrm{PCI}=$ Percutaneous Coronary Intervention

RIMA = Right Internal Mammary Artery

$\mathrm{SAC}=$ Single Aortic Clamp

GSV $=$ Great Saphenous Vein

\section{Introduction}


Neurological complications - specifically stroke - following coronary artery bypass grafting (CABG) operations have been reported with an incidence between 0.8\%-5.2\% [1,2]. Despite the relatively low incidence, the consequences are often devastating $[3,4]$.

Since the occurrence of neurological events dramatically impacts the prognosis and quality of life of the patients, any effort must be pursuit to avoid iatrogenic stroke following CABG. Atherosclerotic disease of the ascending aorta has been considered one of the most probable cause of cerebral embolization due to dislodgement of plaque debris during aortic manipulation and cross-clamping [5-7].

Among the strategies to decrease or eliminate aortic manipulation, off-pump CABG through an aortic "no touch" technique has been advocated as one of the most useful strategy in reducing the stroke rate $[8,9]$, even though it might lead to worse graft patency and survival [10], especially in low-volume Centers [11,12].

However, this off-pump aortic "no touch" technique is not universally applicable and, when saphenous vein and/or free arterial aorto-coronary grafts are used, there is still risk of neurological injury due to tangential aortic clamp applied during the proximal anastomoses sewing.

To minimize aortic manipulation and trauma in standard on-pump CABG, a single aortic clamp (SAC) rather than a double aortic clamp (DAC) has also been proposed, to reduce the manipulation and stretching of the aorta [13-15].

The conclusions of these reports have been conflicting, although in several papers SAC technique showed superiority in reducing neurologic injury following CABG causing less neuropsychological deficits and release of serum S-100 protein, a surrogate marker of cerebral injury [16-18].

On the other hand, SAC prolongs cardiopulmonary bypass (CPB) time, which is also per se considered an independent risk factor for cerebrovascular accidents. Indeed, other papers have reported no benefit of SAC over DAC technique in preventing the neurological lesions [19-21], suggesting that other factors might be accountable for stroke, such as cannulation [22] (cannulation strategy, type of cannula) and even the aortic punch [23].

Given this background, we retrospectively compared the early and long-term neurological outcomes between two homogeneous CABG groups treated with either SAC or DAC technique.

\section{Materials and methods}

\section{Patients}

From March 2006 to December 2012, 251 consecutive patients underwent myocardial revascularization on CPB through a complete median sternotomy performed by a single CABG-experienced surgeon (MA) using SAC or DAC technique. Routine pre-surgical evaluation for all patients included electrocardiogram, echocardiogram, carotid ultrasound and coronary angiography.

\section{Ethical committee}

This work is based on a retrospective chart review with follow-up information retrieved by telephone calls. Patients consent was obtained for research purposes. The informed consent was approved by the local Ethics Committee (CCM 1424), December 2020.

\section{Inclusion criteria}

Inclusion criteria were isolated CABG with no other concomitant cardiac procedures, CPB support, a preoperative left ventricular ejection fraction $(\mathrm{EF})$ more than $50 \%$ and two or more distal coronary anastomoses along with at least one proximal anastomosis on the ascending aorta.

\section{Exclusion criteria}

Exclusion criteria were patients with porcelain aorta or left ventricular mural thrombi identified by preoperative trans-thoracic/trans-oesophageal echocardiogram, angiogram and intraoperative records of atheroma- 
tous aorta (extensive calcifications, focal atheromas) and the presence of clinically relevant internal carotid artery atherosclerosis (stenosis [?]70\% of luminal diameter). Given the exclusion criteria, the analysed patient population is to be considered at low to moderate risk for neurological events.

\section{Surgical procedure}

Anaesthetic protocol included thiopentone, propofol, opiates for induction, sevoflurane, desflurane relaxants, opiates for maintenance and propofol, relaxants and opiates for CPB time. Intraoperative monitoring included radial artery for pressure monitoring and right jugular vein for central venous catheter. Swan-Ganz catheter was not considered as needed as no patient had EF less than 50\%. Urinary catheter and rectal temperature probe were also inserted. All interventions were performed on-pump, $99 \%$ of those through median sternotomy with ascending aorta and right atrial cannulation for CPB implantation, after reaching an activating clotting time more than 480 seconds under systemic heparinization. Only 3 patients (1\%) underwent peripheral femoral cannulation for unstable hemodynamic conditions. In all patients a retrograde cardioplegia catheter was inserted through right atrium directly into coronary sinus. Myocardial protection was obtained using cold hematic blood cardioplegia (Buckberg) administered firstly anterograde and then retrograde every nearly 20 minutes. Patient was cooled to $32-34^{\circ} \mathrm{C}$ during the main time of the operation. The rewarming was achieved with tepid reperfusion and wash-out of the cardioplegia.

The choice of performing a SAC or a DAC was made by the surgeon, depending on the pre-operative imaging tests, on the visual and manipulation appearance of the aorta and on epiaortic ultrasound performed in the operating room. When it appeared to be fibrotic and frail based on the first operator experience, the surgeon preferred a SAC approach.

In case of SAC, the proximal anastomosis was performed during cardiac arrest.

If the surgeon decided to perform DAC, all the grafts were linked to the arterial cannula, so that the coronaries could be perfused immediately after rewarming. After choosing the site of the proximal anastomoses, a Satinsky tangential clamp was placed to allow safe suturing. Subsequently, one by one, the proximal anastomoses were performed.

Total heparinization was antagonized with protamine sulphate after CPB weaning.

\section{Data Collection}

Pre-operative and intra-operative data were collected from clinical records. The main variable investigated was stroke, defined as an acute focal neurological event with a positive magnetic resonance imaging, regardless of duration of clinical symptoms, or a persistent focal neurological deficit lasting longer than 24 hours. Diagnosis of stroke was determined by our cardiac surgical team (at least one cardiac surgeon and one cardiac anesthesiologist) and confirmed by a neurologist in all cases; patients with stroke underwent brain computed tomography and magnetic resonance and the presence of stroke was verified in all patients.

Patients were subsequently followed in our outpatient clinic $(67 \%)$ or through phone calls $(33 \%)$. New neurological and cardiac events (recurrence of angina, acute myocardial infarction, new revascularization procedures, whether percutaneous or surgical) as well as survival data were collected.

\section{Data analysis}

Between-group comparisons for clinical and outcome variables were performed using independent samples t-test, Wilcoxon rank sum test, chi-square analysis, or Fisher's exact test. Data are reported as mean \pm standard deviation, median (first and third quartile), or frequency (\%). All tests are two-sided. Given the exploratory nature of the study, no adjustment for multiple comparisons was performed. Data were compared using the aortic clamping technique (DAC versus SAC as the dichotomizing variable. Analyses were performed using StataV.12 (StataCorp, Texas, US).

\section{Study results}


Patient characteristics are listed in Table 1 . The study cohort included 251 patients. Among them, 217 (86\%) were male and the median age at operation was 68 years (IQR 61-74). Median pre-operative ejection $\mathrm{EF}$ was $60 \%$ (IQR 52-64) and 40 patients (16\%) of the entire cohort had had prior percutaneous coronary intervention (PCI). Patients were divided in two comparable groups (SAC vs. DAC) with no statistically significant difference in demographic characteristics, family history, comorbidities, Euroscore I value and functional class. SAC group included 118 patients while DAC group included 133.

Surgical details are listed in Table 2 . A total of 13 operations (5\%) were emergent surgeries. A total of 11 emergent surgeries (84.61\%) was in the DAC group (p value 0.019), accounting for the $8 \%$ of this cohort.

The grafts used to perform CABG were left internal mammary artery (LIMA) in situ, right internal mammary artery (RIMA) in situ, great saphenous vein (GSV) and left or right arterial artery when GVS was unavailable, always anastomosed to the ascending aorta proximally. Coronary arteries were variably grafted according to the disease as described in Table 2, with no significant difference between the two groups. All distal anastomosis were performed during cardiac arrest (cross-clamping). No difference between the two group was found regarding the number of distal anastomosis, performed either with an arterial ( $p$ value 0.71 ) or a venous graft ( $p$ value 0.071 ). When feasible, a sequential configuration was chosen, performing no more than two bypasses with the same graft.

Single proximal anastomoses were performed in $44(37,2 \%)$ and $61(45,8 \%)$ for the SAC and the DAC groups, respectively $(\mathrm{p}=0,2)$. Double proximal anastomoses were $74(62,8 \%)$ and $72(54,2 \%)$ for the SAC and the DAC groups, respectively.

In the SAC group all the proximal anastomoses were performed during the period of total aortic cross clamping (cardiac arrest). In the DAC group the proximal anastomoses were performed on a beating heart with the aid of a side clamp.

Median surgical time of all cohort and for single group was 240 minutes, with different interquartile ranges for the two groups, 225-300 minutes for DAC and 210-270 minutes for SAC, which account for the statistical significant difference $(p=0.015)$. Median cross-clamp time was longer although not statistically different for the SAC group, 74 minutes (IQR 64-88) vs 72 minutes (IQR 56-84), CPB time was significantly longer in the DAC group, 101 minutes IQR (83-116) vs 88 minutes (IQR 78-103) with a $p<.001$. On the other hand, it's important to highlight that a statistically relevant part of the DAC group (11 patients, $8 \% p=0.019$ ) were referred as emergent surgery and were in worse general conditions, needing longer perfusion CPB time to be weaned off.

We did not found any statistically significant differences in blood loss (median $600 \mathrm{cc}$ IQR 450-750 vs $630 \mathrm{cc}$ IQR 450-850) and blood product use (55 patients -41\%- vs 53 patients - $45 \%^{-}$) within the two groups (DAC vs SAC respectively). Surgical re-exploration for excessive post-operative bleeding was required in 5 patients for each group ( $4 \%$ of the entire cohort). Moreover, no difference in total hours of post-operative invasive ventilation (median of 5 hours IQR 4-7 for each group) neither in total hours of intensive care unit (ICU) stay (mean of $49 \pm 27$ hours) were found. Hospital stay was also comparable with a median of 7 days (IQR 7-9) of total length.

No relevant differences were found in the analysis of peri-procedural complications including the study primary outcome of stroke and transient ischemic attack (Table 3 ). The two groups presented in addition a similar 30-day survival.

Peri-procedural myocardial infarction was overall reported in 7 patients $(3 \%)$, with no difference between the groups. Acute renal failure occurred in 16 patients $(6 \%)$, and $3(1.5 \%)$ needed dialysis assistance. Heart block occurred in 3 patients (1.5\%) while reversible atrial fibrillation in 54 patients $(22 \%)$ of the cohort. We had 5 cases of ischemic stroke $(2 \%)$ with no difference between the two groups $(2-2 \%$ - vs $3-3 \%$ - patients, $P$ .56) while the only case of transient ischemic attack was in the DAC group. No significant differences could be identified. Major Adverse Cerebral and cardiovascular events (MACCE) was 5 (5\%) for the DAC group and $7(6 \%)$ for the SAC group $(\mathrm{p}=0,57)$. Mortality rate at 30 days was $0.5 \%$ accounting for one death in the 
DAC group.

\section{Clinical outcome at follow-up}

\section{Overall Survival}

The follow-up was completed in $82 \%$ of cases and the period ranged from 1 to 172 months ( $127 \pm 34$ months) and from 0,5 to 173 months ( $117 \pm 43$ months) for the SAC and DAC groups, respectively. In the SAC group, a total of 101 patients were included in the follow up analysis. Among them, 31(27,9\%) died: 14/31(45\%) for a cardiac cause, 2/31(6.4\%) for respiratory insufficiency, 8/31(25.8\%) for extracardiac cause and in the remaining 7 cases $(22,6 \%)$ the cause of death remained unknown because of a non-retrievable information.

In the DAC group (104 patients), 29(27,9\%) died: 6/29(20,7\%) for a cardiac cause, 3/29(10,3\%) for extracardiac cause, $20 / 29(68,9 \%)$ the cause of death remained unknown.

The all-cause mortality Kaplan-Meier estimates show $81 \%$ and $73 \%$ of survival, for DAC and SAC respectively, at a median follow-up of 11 years(Fig.1.A).

\section{Stroke and MACCE}

For the SAC group, 1 patient $(0,9 \%)$ experienced recurrent myocardial angina during follow-up, five patients $(4.9 \%)$ showed acute myocardial infarction and only 1 patient $(0.9 \%)$ had stroke. Freedom from MACCE defined as myocardial infarction, stroke and cardiac death, was 100\%, 100\%, 95\% and $42 \%$ at 1,5,10 and 14 years respectively (Fig.1B).

For the DAC group, 4 patients (3,4\%) experienced recurrent myocardial angina and 2 patients (2\%) showed acute myocardial infarction, no patient experienced stroke at follow up. Freedom from MACCE defined as myocardial infarction, stroke and cardiac death, was $100 \%, 100 \%, 98 \%$ and $82 \%$ at 1,5, 10 and 14 years respectively (Fig.1B). The difference between $\mathrm{SAC}$ and DAC is statistically significant ( $\mathrm{p}$ value $=0.02$ ). The two groups have similar incidence of MACCE in the first 10 years (fig 1.C) ( $p$ value $=0.2465$ ), on the other hand, after 10 years, the two curves diverge and become significantly different (fig.1.D) (p value $=0.043$ ).

Therefore, at the chi-squared analysis, after normalizing for presence of diabetes and peripheral arterial diseases, being part of the SAC group was a risk factor for having MACCE at follow up (p value $=0.035$, HR 5,46 95\% CI $1.14-26.1$ ).

\section{Discussion}

We presented a retrospective analysis of the differences in neurological outcomes related to the clamping approach (single vs double SAC and DAC respectively) of the aorta when performing CABG in a low to moderate risk population. A single, experienced surgeon performed all the procedures. A total of 5 patients $(2 \%)$ had stroke with no significant clinical sequalae, probably because of the limited interested cerebral anatomical area. We could not be able to find any significant difference in the stroke rate between the SAC and DAC group.

Classically, many risks factors have been linked to stroke following CABG. First, the use of CPB has been advocated to be related with a certain risk of neurocognitive sequelae linked with inflammatory response and microembolism [4-9, 24].

In our series, all patients underwent on-pump CABG, even if in the literature there is still an on-going debate between the usefulness of on-pump versus off-pump coronary artery bypass (OPCAB) surgery. Prospective studies like ROOBY trial showed better results in term of myocardial infarction (lower rate in on-pump CABG) and better rate of venous graft patency at 1 year, along with better Fitz-Gibbon grade, for on-pump CABG [25]. While other studies such as the SMART trial [26] highlighted no significant differences between the two techniques in term of mortality, myocardial infarction, stroke and recurrent angina or readmission for cardiac or non-cardiac events. Regarding neurological outcomes, The ROOBY trial [25] showed a better scoring in the clock-drawing test in the off-pump group. 
Therefore, there must be other variables linked to peri-operative stroke following CABG. Indeed, cannulation itself (including type of cannula), cross-clamping and, more widely, aortic manipulation, are \soutshowed suspected to be linked with neurocognitive impairment [12-14].

The debate regarding the best strategy for proximal anastomosis (SAC vs DAC) is still on-going. Chu D. et al.[27] performed a retrospective study on 1819 patients without finding any difference in terms of neurological events between SAC and DAC patients. Araque JC et al.[20] had the same results on a bigger cohort of patients and, moreover, at the univariate and multivariate analysis the degree of aortic manipulation was not associated with post-operative stroke. Their results were confirmed by a large, retrospective study on patients recruited from the Society of Thoracic Surgery database; no differences between SAC and DAC were found regarding neurological events and mortality [28].

On the other hand, several authors have showed conflicting results. Remarkably, Hammon et al.[18] prospectively analyzed 237 high risk patients undergoing OPCABG vs. CABG with single vs multiple clamp technique and failed to find an improve outcome in term of neuropsychological deficit in the OPCAB group. More frequent neuro-psychological deficits were found in the DAC group. Instead, patients undergoing CABG with single clamping had better outcome, suggesting that the mild hypothermia during CPB may allow a neuro-protective environment [29].

In our cohort none of the techniques used showed to be superior in term of stroke incidence over the other. As each technique has its own surgical advantages and disadvantages (for instance, more space to perform proximal anastomosis in SAC and direct evaluation of graft's length in DAC) the surgeon should choose the technique most appropriate to a specific situation, given the fact that good clinical outcomes and low complication rates could be achieved with both techniques. However, according to the existing evidences, the presence of aortic atheromas, fibrosis or frail tissue push the operator to reduce aortic manipulation to reduce the risk of local or distal problems, and thus a SAC strategy could be preferred over DAC. OPCABG and use of double mammary graft or Y configuration could be advocated in the case of porcelain aorta to avoid excessive aortic manipulation.

On this regard an interesting aspect, as reported by Hammon et al.[18], is that neuropsychological deficits, even if absent and even not radiologically detectable early after operation, can appear over a period of six months from the index procedure, suggesting that a closer neurological follow-up should be taken into consideration in high-risk patients to better estimate the real neurological outcome .

Our study offers a very long follow-up, reaching up to 14 years. In the SAC group, 1 (0.9\%) patient had stroke during follow up, while in the DAC group nobody had neurological events. Therefore, it is realistic to assume that most of the strokes after CABG happen in the early post-operative period, and that the risks related to aortic manipulation decrease in the long-term period.

Finally, it is interesting to notice that after 10 years the SAC group had a significant lower freedom from MACCE, often related to the progression of the vasculopathy. Indeed, patients in the SAC group had a 5-times higher risk of MACCE than patients in the DAC group during long-term follow-up.

This result further underlines that an accurate analysis of the aorta at the time of surgery may reveal many pathological changes that put the patients at risk, immediately and during follow-up. Therefore, besides pre-operative imaging exams and anamnesis, a in-depth analysis of the features and consistency of the aorta at the time of surgery is crucial to decide if a DAC or a SAC is the best strategy. It is possible to assume that our patients in the SAC group had a worse vascular tissue already at time of CABG, and this would explain the higher incidence of MACCE at follow up. Hence, patients who have a worse aortic tissue should be kept in a strict long-term follow up (up to 10 years) for MACCE.

\section{Study limitation}

The main limitation of this paper is related on its retrospective design analysis on a single-operator outcome. The main bias is the analysis of pre-operatively judged low neurological risk patients, thus our conclusion could not be applicable to other subset of CABG patients. Moreover, the small sample size of patients who 
were able to perform a follow up is another limitation. Finally, a last bias of our study is the assessment of the single surgeon conducted on the aorta of the patients that led him to choose between SAC and DAC.

\section{Conclusion}

In conclusion, incidence of stroke seems to be independent from cross-clamping technique in a non-severely calcified aorta with low to moderate carotid atherosclerotic burden.

More generally, we could infer that the global rate of stroke after CABG is probably more influenced by other factors (such as, for example, plaques extent and composition).

We do believe in the principle of knowing and respecting the aorta: a careful pre-operative and intraprocedural analysis of the aorta and of the supra-aortic vessels is the best strategy to personalize the surgical therapy to achieve optimal general and neurological outcomes.

Acknowledgement :we state no acknowledgement.

Author contributions statement: Giorgio Mastroiacovo:writing-original draft, data curation, Marco Gennari:writing-original draft, data curation, Ilaria Giambuzzi:writing-original draft, data curation, Sergio Pirola:conceptualization, Giuseppe Nanci: data curation, Alice Bonomi: formal analyses, Gabriele Egidy Assenza : data curation, Marco Agrifoglio : Investigation, supervision.

Funding statement :we state no funding source for this work

Conflict of interest statement :none related to this paper.

\section{List of Figure:}

Fig.1:A: All-cause mortality;B: MACCE; C: All-cause mortality (first 10 years);D: All-cause mortality ( after 10 years).

\section{References}

1. Stamou SC,Hill PC,Dangas G,Pfister AJ,Boyce SW,Dullum MK,et al. Stroke after coronary artery bypass: incidence, predictors, and clinical outcome. Stroke. 2001;32:1508-1513.

2. Tarakji KG,Sabik JF,Bhudia SK,Batizy LH,Blackstone EH. Temporal onset, riskfactors, and outcomes associated with stroke after coronary artery bypass grafting. JAMA. 2011;305:381-390.

3. Mehta A,Gleason T,Wechsler L,Winger D, Wang L, Thirumala PD. Perioperative stroke as a predictor of mortality and morbidity in patients undergoing CABG. J Clin Neurosci. 2017 Oct;44:175-179.

4. Dacey LJ,Likosky DS,Leavitt BJ,Lahey SJ,Quinn RD,Hernandez F Jr, et al. Northern New England Cardiovascular Disease Study Group. Perioperative stroke and long-term survival after coronary bypass graft surgery. Ann Thorac Surg. 2005 Feb;79(2):532-6.

5. Selnes OA,Goldsborough MA,Borowicz LM,McKhann GM. Neurobehavioural sequelae of cardiopulmonary bypass. Lancet. 1999;353:1601-6.

6. Fearn SJ,Pole R,Burgess SG,Ray TL,Hooper CN,McCollum CN. Cerebral embolization during modern cardiopulmonary bypass. Eur J Cardiothorac Surg 2001;20:1163-7.

7. Barbut D,Hinton RB,Statrowski TP,et al. Cerebral emboli detected during bypass surgery are associated with clamp removal. Stroke 1994;25:2398-402.

8. Van der Linden J,Casimir-Ahr H. When do cerebral emboli appear during open heart operations? A transcranial Doppler study. Ann Thorac Surg. 1991;51:237-41.

9. Patel NC,Pullan MD,Fabri BM. Does off-pump total arterial revascularization without aortic manipulation influence neurological outcome? A study of 226 consecutive, unselected cases. Heart Surg forum 2001,5:28-32.

10. Hattler B.,Messenger J.C.,Shroyer A.L.,Collins J.F.,Haugen S.J.,Garcia J.A. et al. Off-pump coronary artery bypass surgery is associated with worse arterial and saphenous vein graft patency and less effective revascularization: results from the veterans affairs randomized on/off bypass (ROOBY) trial. Circulation. 2012; 125: 2827-2835 
11. Taggart DP,Gaudino MF,Gerry S,Gray A,Lees B,Sajja LR,et al. Arterial Revascularization Trial Investigators. Ten-year outcomes after off-pump versus on-pump coronary artery bypass grafting: Insights from the Arterial Revascularization Trial. J Thorac Cardiovasc Surg. 2020 Feb 19:S0022-5223(20)30427$\mathrm{X}$.

12. Kim KB,Kang CH,Chang WI,et al. Off-pump coronary artery bypass with complete avoidance of aortic manipulation. Ann Thorac Surg. 2002;74(Suppl):S1377-82.

13. Leacche M,Carrier M,Bouchard D, et al. Improving neurological outcome in off-pump surgery : the "no touch" technique. Heart Surg forum 2003,6:169-75.

14. Kapetanakis EI,Stamou SC,Dullum MK,Hill PC,Haile E,Boyce SW,et al. The impact of aortic manipulation on neurologic outcomes after coronary artery bypass surgery: a risk-adjusted study. Ann Thorac Surg. 2004 Nov;78(5):1564-71.

15. Dar MI,Gillot T,Ciulli F,Cooper GJ. Single aortic cross-clamp technique reduces S-100 release after coronary artery surgery. Ann Thorac Surg. 2001;71:794-6.

16. Lorusso R,Moscarelli M,Di Franco A,Grazioli V,Nicolini F,Gherli T,et al. Association Between Coronary Artery Bypass Surgical Techniques and Postoperative Stroke. J Am Heart Assoc. 2019 Dec 17;8(24):e013650.

17. Daniel WT,Kilgo P,Puskas JD,Thourani VH,Lattouf OM,Guyton RA et al. Trends in aortic clamp use during coronary artery bypass surgery: effect of aortic clamping strategies on neurologic outcomes. J Thorac Cardiovasc Surg. 2014 Feb;147(2):652-7.

18. Hammon JW,Stump DA,Butterworth JF, et al. Coronary artery bypass grafting with single cross-clamp results in fewer persistent neuropshychological deficits than multiple clamp or off-pump coronary artery bypass grafting. Ann Thorac Surg 2007;84:1174-9.

19. Chu D,Schaheen L,Morell VO,Gleason TG,Cook CC,Wei LM et al. Effect of Aortic Clamping Strategy on Postoperative Stroke in Coronary Artery Bypass Grafting Operations. JAMA Surg. 2016 Jan;151(1):59-62.

20. Araque JC, Greason KL, Li Z, Heins CN, Stulak JM, Daly RC, et al. On-pump coronary artery bypass graft operation: Is one crossclamp application better than two? J Thorac Cardiovasc Surg. 2015 Jul;150(1):145-9.

21. Alaeddine M,Badhwar V,Grau-Sepulveda MV,Wei LM,Cook CC,Halkos ME,et al. Aortic clamping strategy and postoperative stroke. J Thorac Cardiovasc Surg. 2018 Oct;156(4):1451-1457.e4.

22. Grocott H.P,Croughwell N.D.,Amory D.W.,White W.D.,Kirchner J.L.,Newman M.F. Cerebral emboli and serum S100B during cardiac operations. Ann Thorac Surg. 1998; 65: 1645-1650

23. Kim RW,Mariconda DC,Tellides G,Kopf GS,Dewar ML,Lin Z, et al. Singleclamp technique does not protect against cerebrovascular accident in coronary artery bypass grafting. Eur J Cardiothorac Surg. 2001;20:127-32.

24. Gottesman RF,Wityk RJ. Brain injury from cardiac bypass procedures. Semin Neurol. 2006 Sep;26(4):432-9.

25. Shroyer AL,Grover FL,Hattler B,Collins JF,McDonald GO,Kozora E,et al. Veterans Affairs Randomized On/Off Bypass (ROOBY) Study Group. On-pump versus off-pump coronary-artery bypass surgery. N Engl J Med. 2009 Nov 5;361(19):1827-37.

26. Puskas JD,Williams WH,Mahoney EM,Huber PR,Block PC,Duke PG, et al. Off-pump vs conventional coronary artery bypass grafting: early and 1-year graft patency, cost, and quality-of-life outcomes: a randomized trial. JAMA. 2004 Apr 21;291(15):1841-9.

27. Chu D,Schaheen L,Morell VO,Gleason TG,Cook CC, et al. Effect of Aortic Clamping Strategy on Postoperative Stroke in Coronary Artery Bypass Grafting Operations. JAMA Surg. 2016 Jan;151(1):5962.

28. Mohamad Alaeddine,Vinay Badhwar,Maria V Grau-Sepulveda ,Lawrence M Wei ,Chris C Cook,et al. Aortic clamping strategy and postoperative stroke J Thorac Cardiovasc Surg 2018 Oct;156(4):14511457.e4.

29. Raja SG,Navaratnarajah M,Fida N,Kitchlu CS. For patients undergoing coronary artery bypass grafting at higher risk of stroke is the single cross-clamp technique of benefit in reducing the incidence of 
stroke? Interact Cardiovasc Thorac Surg 2008;7:500-3.

\section{Hosted file}

Table 1.pdf available at https://authorea.com/users/316050/articles/509470-cross-clamptechnique-and-incidence-of-stroke-after-coronary-artery-bypass-grafting-know-andrespect-the-aorta-principle

\section{Hosted file}

Table 2.pdf available at https://authorea.com/users/316050/articles/509470-cross-clamptechnique-and-incidence-of-stroke-after-coronary-artery-bypass-grafting-know-andrespect-the-aorta-principle

\section{Hosted file}

Table 3.pdf available at https://authorea.com/users/316050/articles/509470-cross-clamptechnique-and-incidence-of-stroke-after-coronary-artery-bypass-grafting-know-andrespect-the-aorta-principle

A

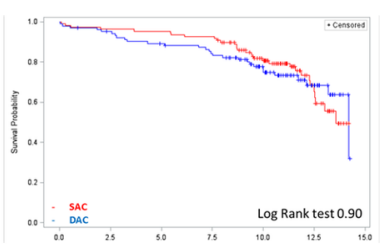

$\mathrm{C}$

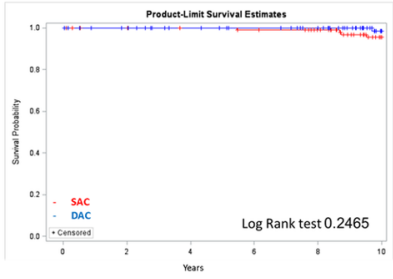

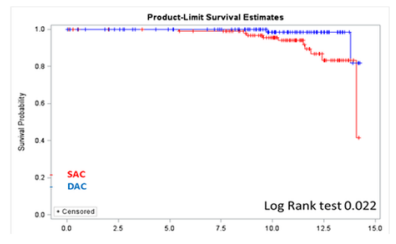

D

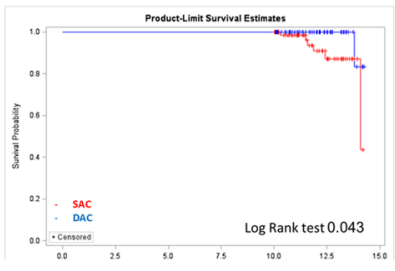

